\title{
Microarray expression profile of long non-coding RNAs in EGFR-TKIs resistance of human non-small cell lung cancer
}

\author{
NINGNING CHENG ${ }^{1}$, XUEFEI LI $^{2}$, CHAO ZHAO $^{2}$, SHENGXIANG REN $^{1}$, XIAOXIA CHEN $^{1}$, WEIJING CAI ${ }^{1}$, \\ MINGCHUAN ZHAO ${ }^{1}$, YISHI ZHANG ${ }^{1}$, JIAYU LI ${ }^{1}$, QI WANG $^{1}$ and CAICUN ZHOU ${ }^{1}$ \\ Departments of ${ }^{1}$ Medical Oncology, and ${ }^{2}$ Lung Cancer and Immunology, Shanghai Pulmonary Hospital, \\ Tongji University, Tongji University Medical School Cancer Institute, Shanghai 200433, P.R. China
}

Received July 23, 2014; Accepted November 7, 2014

DOI: $10.3892 /$ or.2014.3643

\begin{abstract}
The application of epidermal growth factor receptor (EGFR) tyrosine kinase inhibitors (TKIs) is limited by drug resistance in non-small cell lung cancer (NSCLC). Long non-coding RNAs (lncRNAs) are known to be involved in tumor development and metastasis, as well as chemotherapy resistance. To gain insight into the molecular mechanisms of EGFR-TKIs resistance, EGFR-TKIs-sensitive and -resistant human lung cancer cells were analyzed by lncRNA microarray. In the present study, we found a total of 22,587 lncRNAs expressed in lung cancer cells. Of these, the expression level of 1,731 lncRNAs was upregulated $>2$-fold compared with gefitinib-sensitive cells while that of 2,936 was downregulated. Bioinformatics analysis (GO and pathway analyses) revealed that some classical pathways participating in cell proliferation and apoptosis were aberrantly expressed in these cells (P-value cut-off was 0.05). Enhancer-like lncRNAs and their nearby coding genes were analyzed. Six lncRNAs were identified as potential enhancers. Several lncRNAs were validated in lung cancer cell lines using RT-qPCR. To the best of our knowledge, the results showed for the first time that differentially expressed lncRNAs responded to EGFR-TKIs resistance in NSCLC cells. LncRNAs may therefore be novel candidate biomarkers and potential targets for EGFR-TKIs therapy in the future.
\end{abstract}

\section{Introduction}

Non-small cell lung cancer (NSCLC) patients harboring activating mutations in epidermal growth factor receptor (EGFR)

Correspondence to: Dr Caicun Zhou, Department of Medical Oncology, Shanghai Pulmonary Hospital, Tongji University, Tongji University Medical School Cancer Institute, No. 507 Zhengmin Road, Shanghai 200433, P.R. China

E-mail: caicunzhou@gmail.com

Key words: long non-coding RNAs, non-small cell lung cancer, EGFR-TKIs, drug resistance initially respond well to treatment with EGFR-tyrosine kinase inhibitors (TKIs) such as gefitinib or erlotinib $(1,2)$. However, drug resistance to treatment with EGFR-TKIs eventually is inevitable and limits the clinical benefits. The mechanisms underlying EGFR-TKIs resistance are multi-factorial and many have been reported, for example, the T790M mutation in EGFR, MET amplification, $N F-\kappa B$ activation and emergence of SCLC (3-5). However, $30 \%$ resistant mechanisms are unknown. Thus, elucidating the molecular mechanisms underlying EGFR-TKIs resistance is essential for the identification of key biomarkers.

Recent findings have shown that non-coding RNAs (ncRNAs) are involved in the pathogenesis of NSCLC, providing new insights into the biology of diseases (6-9). Long non-coding RNAs (lncRNAs) are non-protein coding transcripts, >200 nucleotides (nt) in length. IncRNAs play important regulatory roles in the cancer development, metastasis and chemotherapy resistance of multiple types of cancer (10-12). Accumulating evidence suggested that lncRNAs are abnormally expressed in various types of human cancer (10,13-15). Studies have reported that lncRNAs influence chemotherapy resistance (16-18). For example, IncRNA-AK126698 is associated with cisplatin resistance in NSCLC (19). Another study has shown that cisplatin-based chemotherapy results in the upregulation of UCA1 expression in patients with bladder cancer (17). Therefore, lncRNAs are possible novel candidate biomarkers and potential targets for EGFR-TKIs therapy.

To ascertain whether IncRNAs expression signatures can differ between gefitinib-sensitive PC9 and gefitinibresistant PC9/R cells, lncRNAs expression profiles were identified using microarray analysis. Subsequent to obtaining expression-profile results for these samples, we investigated the relationship between $\operatorname{lncRNAs}$ and EGFR-TKIs resistance in NSCLC.

\section{Materials and methods}

Cell culture. PC9 (EGFR exon 19 deletion), H1975 (L858R/ T790M), H1299 and A549 (EGFR wild-type) human lung adenocarcinoma cell line were obtained from the American Type Culture Collection (ATCC; Vanassas, MA, USA). Gefitinib-induced acquired resistant lung cancer cells from PC9 (PC9/R) were provided by the Shanghai Pulmonary 
Hospital. The cells were cultured in a $37^{\circ} \mathrm{C}$ humidified incubator with $5 \% \mathrm{CO}_{2}$ in Dulbecco's modified Eagle's medium (DMEM) supplemented with $10 \%$ fetal bovine serum (FBS) (both from Gibco Life Technologies, Carlsbad, CA, USA).

Cell proliferation assay. Cells were seeded in 96-well plates at $5 \times 10^{3}$ cells/well, and treated with various concentration of gefitinib for $72 \mathrm{~h}$. At the end of incubation, the cell proliferation reagent Cell Counting Kit-8 (CCK-8; Dojindo Laboratories, Japan) $(10 \mu \mathrm{l})$ was added to each well and incubated for $1 \mathrm{~h}$ at $37^{\circ} \mathrm{C}$. Viable cell numbers were estimated by measurement of optical density (OD) at $450 \mathrm{~nm}$.

LncRNA microarray. The Arraystar Human LncRNA Microarray v2.0 has been designed for the global profiling of human lncRNAs and protein-coding transcripts. A total of 33,045 lncRNAs and 30,215 coding transcripts can be detected by second-generation lncRNA microarray. The lncRNAs were carefully collected from the most authoritative databases, including RefSeq, UCSC knowngenes, Ensembl and a number of related studies. Each transcript is represented by a specific exon or splice junction probe that can accurately identify individual transcripts. Positive probes for housekeeping genes and negative probes were also printed onto the array for hybridization quality control.

RNA labeling and array hybridization. Sample labeling and array hybridization were performed according to the Agilent One-Color Microarray-Based Gene Expression Analysis protocol (Agilent Technologies, Inc., Santa Clara, CA, USA) with minor modifications. Briefly, mRNA was purified from total RNA following removal of rRNA (mRNA-ONLY ${ }^{\mathrm{TM}}$ Eukaryotic mRNA Isolation kit; Epicentre). Each sample was amplified and transcribed into fluorescent cRNA along the entire length of the transcripts without 3 ' bias utilizing a random priming method. The labeled cRNAs were purified using an RNeasy Mini kit (Qiagen, Valencia, CA, USA). The concentration and specific activity of the labeled cRNAs (pmol Cy3/ $\mu$ g cRNA) were measured by NanoDrop ND-1000. Each labeled cRNA $(1 \mu \mathrm{g})$ was fragmented by adding $5 \mu \mathrm{l} 10 \mathrm{X}$ blocking agent and $1 \mu \mathrm{l}$ of $25 \mathrm{X}$ fragmentation buffer. The mixture was heated at $60^{\circ} \mathrm{C}$ for $30 \mathrm{~min}$, and $25 \mu \mathrm{l} 2 \mathrm{X} \mathrm{GE}$ hybridization buffer was added to dilute the labeled cRNA. Hybridization solution $(50 \mu \mathrm{l})$ was dispensed into the gasket slide and assembled to the IncRNA expression microarray slide. The slides were incubated for $17 \mathrm{~h}$ at $65^{\circ} \mathrm{C}$ in an Agilent hybridization oven. The hybridized arrays were washed, fixed and scanned using the Agilent DNA Microarray Scanner (part no. $\mathrm{G} 2505 \mathrm{C})$.

RNA extraction and quantitative PCR. Total RNA was extracted from lung cancer cell lines using TRIzol reagent (Takara, Japan). The expression of lncRNAs in lung cancer cell lines was measured by qPCR using SYBR Premix Ex Taq (Takara code: DRR420A) on MX3000P instrument and using the following cycling parameters: initial denaturation at $95^{\circ} \mathrm{C}$ for $30 \mathrm{sec}$, followed by 40 cycles of $95^{\circ} \mathrm{C}$ for $5 \mathrm{sec}, 60^{\circ} \mathrm{C}$ for $20 \mathrm{sec}$ and $95^{\circ} \mathrm{C}$ for $60 \mathrm{sec}$, and $60^{\circ} \mathrm{C}$ for $30 \mathrm{sec}$. Primers were designed by Sangon Biotech (China). GAPDH was used as a control. Experiments were carried out in triplicate. The median

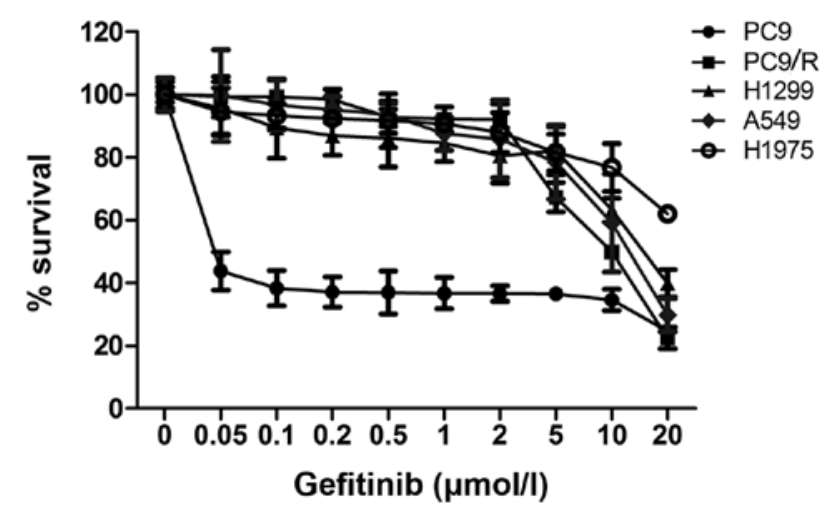

Figure 1. Cells were treated with various concentrations of gefitinib. After $72 \mathrm{~h}$, cell survival was measured. Data in histograms are means $\pm \mathrm{SD}$. $\mathrm{P}<0.01$ (t-test), compared with PC9.

Table I. The differentially expressed lncRNAs in gefitinib-resistant and gefitinib-sensitive lung cancer cells.

\begin{tabular}{lccc}
\hline $\begin{array}{l}\text { Up- or } \\
\text { Downregulated }\end{array}$ & $\begin{array}{c}\text { Fold-change } \\
>2\end{array}$ & $\begin{array}{c}\text { Fold-change } \\
>5\end{array}$ & $\begin{array}{c}\text { Fold-change } \\
>10\end{array}$ \\
\hline Up & 1,731 & 79 & 6 \\
Down & 2,936 & 326 & 43 \\
\hline
\end{tabular}

lncRNAs, long non-coding RNAs.

in each triplicate was used to calculate relative lncRNA concentrations using the formula: $\Delta \mathrm{Ct}=\mathrm{Ct}_{\text {median IncRNAs }}-\mathrm{Ct}_{\text {median GAPDH }}$. Expression fold changes were calculated using $2^{-\Delta \Delta \mathrm{Ct}}$ methods.

Data analysis. The Agilent Feature Extraction software (version 11.0.1.1) was used to analyze acquired array images. Quantile normalization and subsequent data processing were performed using the GeneSpring GX v11.5.1 software package (Agilent Technologies). Differentially expressed LncRNAs and mRNAs were identified through fold-change filtering. Hierarchical clustering was performed using the Agilent GeneSpring GX software (version 11.5.1). Gene ontology (GO) and pathway analysis were performed in the standard enrichment computation method.

Statistical analysis. Statistical analysis was performed using SPSS version 17.0 software (SPSS, Inc., Chicago, IL, USA). Results were presented as means \pm standard deviation (SD) of three separate assays. Differences between groups were assessed using the t-test (two-tailed). $\mathrm{P}<0.05$ was considered to indicate a statistically significant result.

\section{Results}

Differentially expressed lncRNAs and mRNAs. Gefitinibresistant PC $9 / \mathrm{R}$ cells were identified by evaluating the $\mathrm{IC}_{50}$ value of PC9/R against the PC9 cell line. The $\mathrm{IC}_{50}$ value of gefitinib for the drug resistant PC9/R cell line was $15 \mu \mathrm{mol} / 1$ was 300 -fold higher than that of the PC9 cell line $(0.05 \mu \mathrm{mol} / \mathrm{l})$ 
A

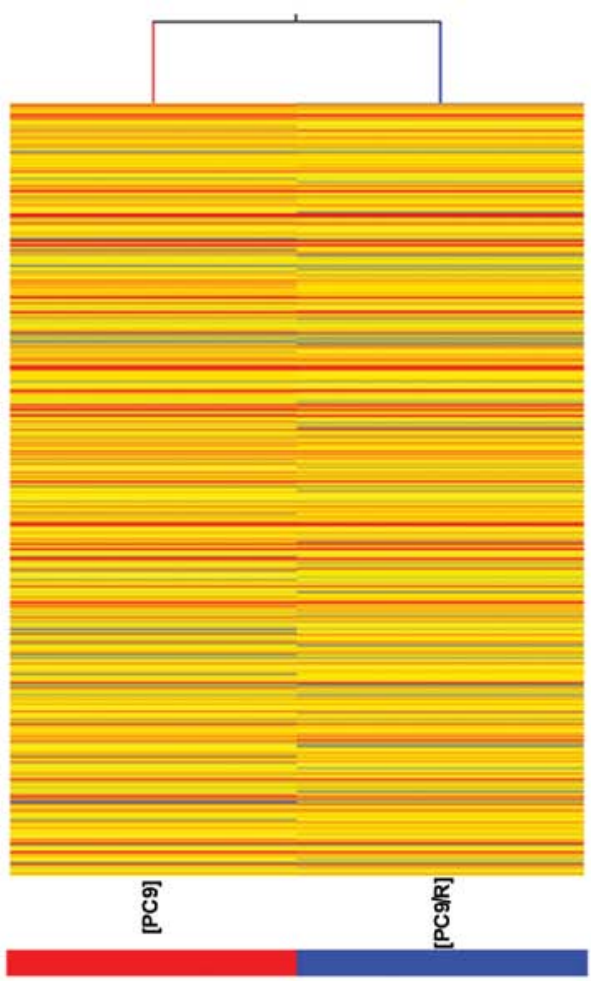

C

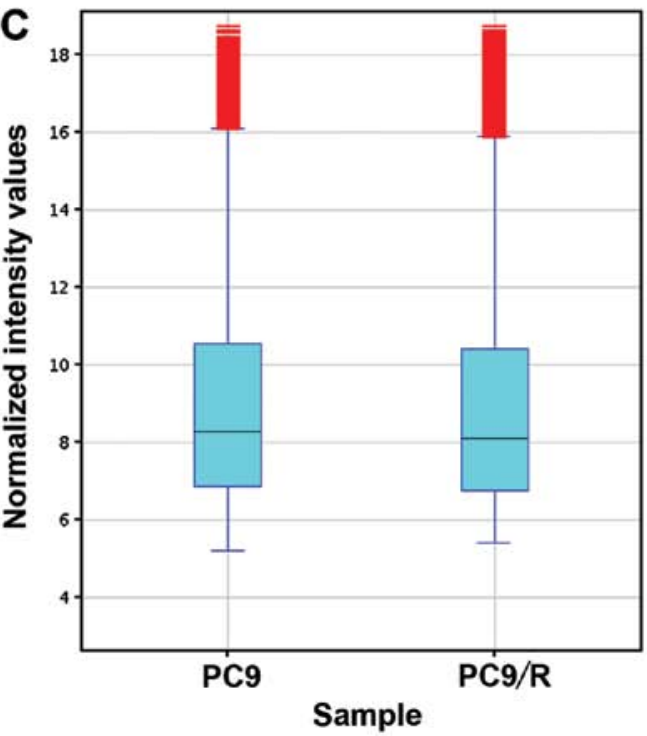

B

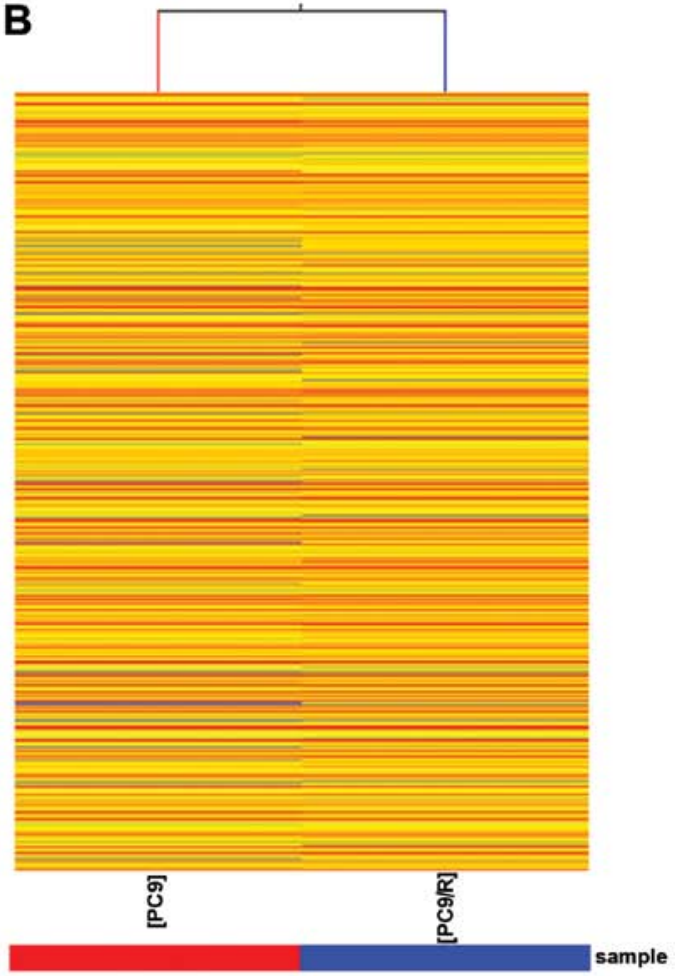

D

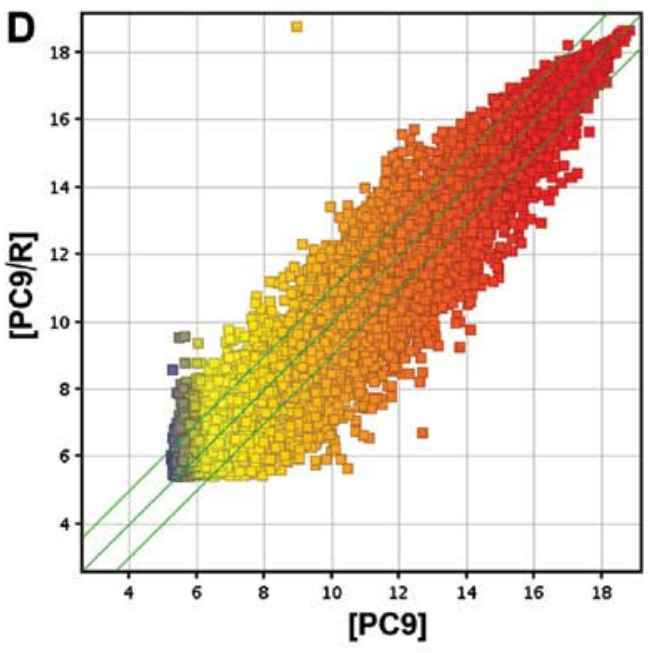

$\begin{array}{ll}\mathrm{X} \text {-axis }[\mathrm{PC9}] & \mathrm{V} \\ \mathrm{Y} \text {-axis }[\mathrm{PC9} / \mathrm{R}] & \mathrm{v}\end{array}$

Figure 2. RNA expression profiles in PC9 and PC9/R cells. Hierarchical clustering indicates (A) lncRNA and (B) mRNA profiles. (C) Box plot is a traditional method for visualizing the distribution of a dataset. (D) Scatter plot is a visualization that is useful for assessing the variation between chips. 'Red' shows a high relative expression, and 'blue' a low relative expression. The values of the $\mathrm{x}$ - and $\mathrm{y}$-axes in the scatter plot are the average normalized signal values of the group ( $\log 2$ scaled). PC9/R, gefitinib-induced acquired-resistant lung cancer cells derived from PC9; lncRNAs, long non-coding RNAs.

(Fig. 1). This result demonstrated that PC9/R cells were more resistant to gefitinib than PC9 cells.

For the microarray analysis, 22,587 lncRNAs (Fig. 2A, $\mathrm{C}$ and D) and 17,479 mRNAs (Fig. 2B) were differentially expressed in PC9 and PC9/R with gefitinib-sensitive and acquired-resistant cells, respectively. Of these, 1,731 lncRNAs were upregulated $>2$-folds in PC9/R compared to PC9 cells, while 2,936 were downregulated $(\mathrm{P}<0.01)$ (Table I). In addition, as compared to PC9 cells, 2,349 mRNAs were increased and 1,307 were decreased in the expression level in $\mathrm{PC} 9 / \mathrm{R}$ cells (fold-change $>2, \mathrm{P}<0.01$ ).
GO analysis. GO analysis is a functional analysis associating differentially expressed mRNAs with GO categories. In the present study, GO analysis was performed to determine the gene and gene product enrichment. The Fisher's exact test was used to determine whether there was more overlapping between the $\mathrm{DE}$ and the GO annotation list than would be expected by chance.

We found that the highest enriched GOs targeted by upregulated transcripts were cellular metabolic process (GO: biological processes), intracellular component (GO: cellular components), and protein binding (GO: molecular func- 
Table II. Gene ontology categories ranked by cell proliferation and apoptosis.

\begin{tabular}{llrrr}
\hline GO.ID & \multicolumn{1}{c}{ Term } & Count & Fold. enrichment & P-value \\
\hline $\begin{array}{l}\text { Upregulated } \\
0006915\end{array}$ & Apoptotic process & & & \\
0008283 & Cell proliferation & 216 & 1.252336427 & 0.000174419 \\
0006917 & Induction of apoptosis & 190 & 1.181451791 & 0.00654506 \\
Downregulated & & 56 & 1.259490591 & 0.040513474 \\
0097190 & Apoptotic signaling pathway & & & \\
2001233 & Regulation of apoptotic signaling pathway & 7 & 2.872964169 & 0.008043735 \\
0097193 & Intrinsic apoptotic signaling pathway & 4 & 3.201302932 & 0.032869143 \\
0008283 & Cell proliferation & 124 & 1.410666537 & $4.12154 \mathrm{E}-05$ \\
0042127 & Regulation of cell proliferation & 94 & 1.415439678 & 0.000335816
\end{tabular}

GO, gene ontology.

\section{A}
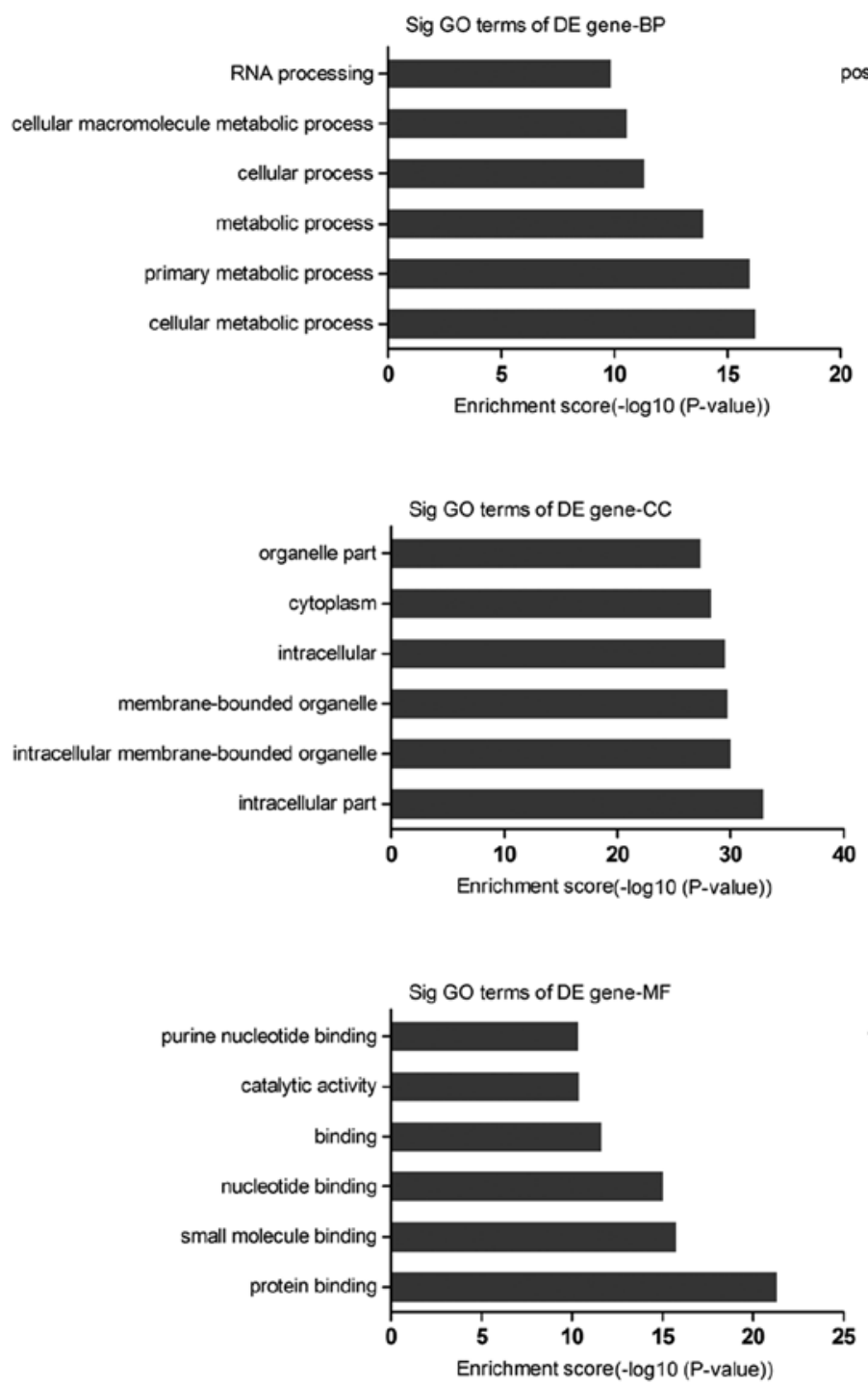

B

\section{GO down-regulated}
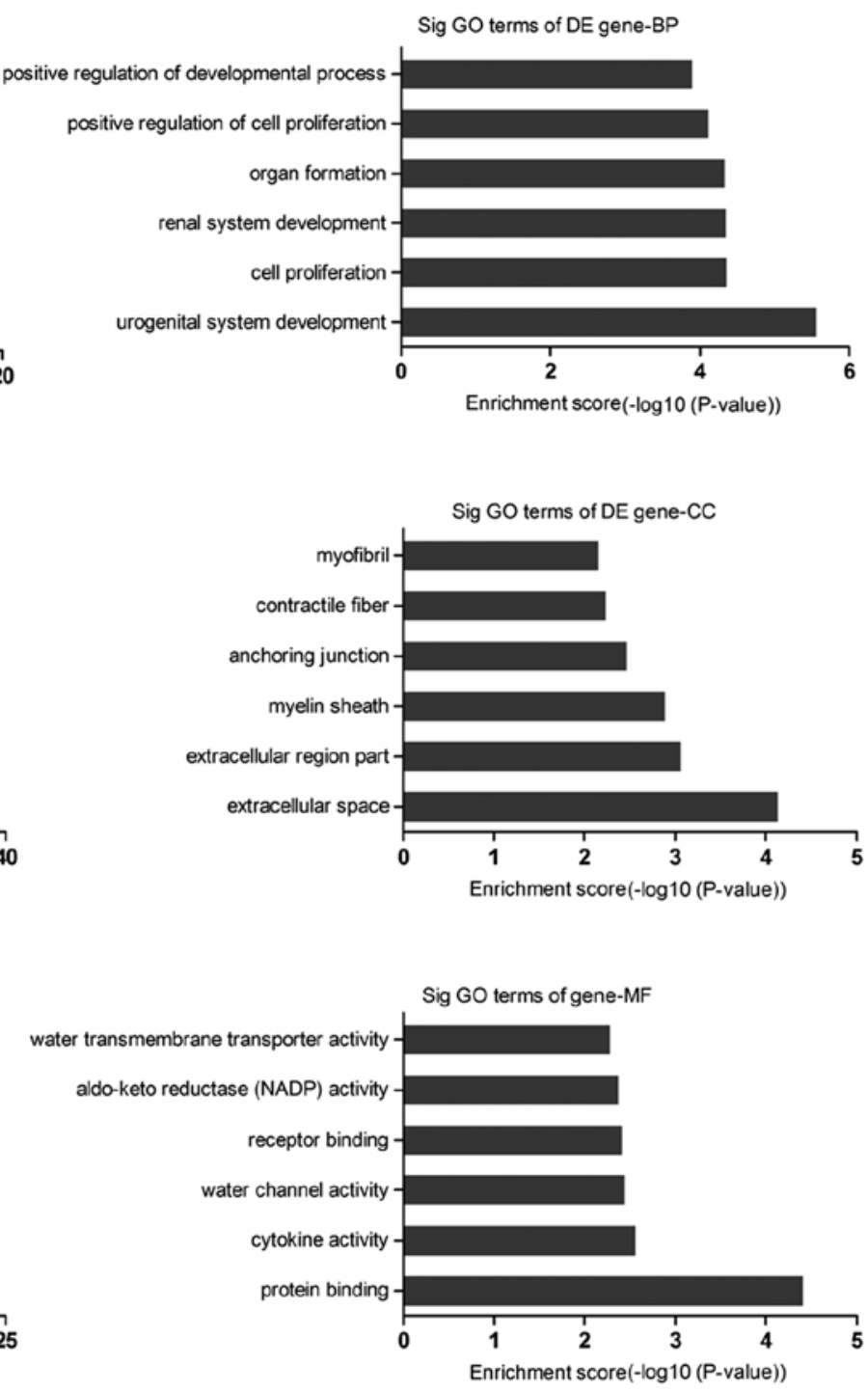

Figure 3. The Gene ontology (GO) project provides a controlled vocabulary to describe gene and gene product attributes in any organism. (A) GO upregulated. (B) GO downregulated. The P-value denotes the significance of GO terms enrichment in the DE genes. The lower the P-value, the more significant the GO term ( $\mathrm{P} \leq 0.05$ was recommended as the cut-off value). 


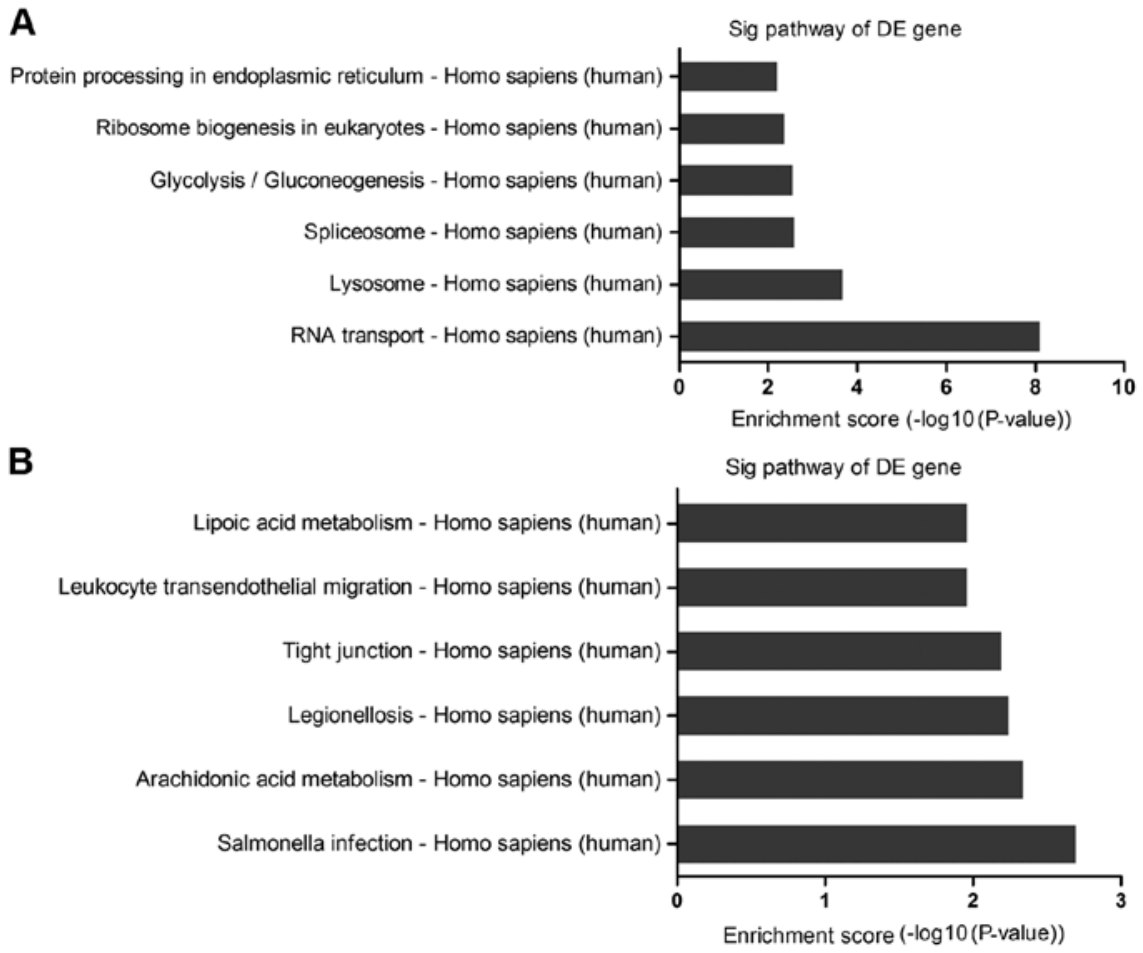

Figure 4. Signaling pathways of differentially expressed upregulated (A) mRNAs and downregulated (B) mRNAs.

A

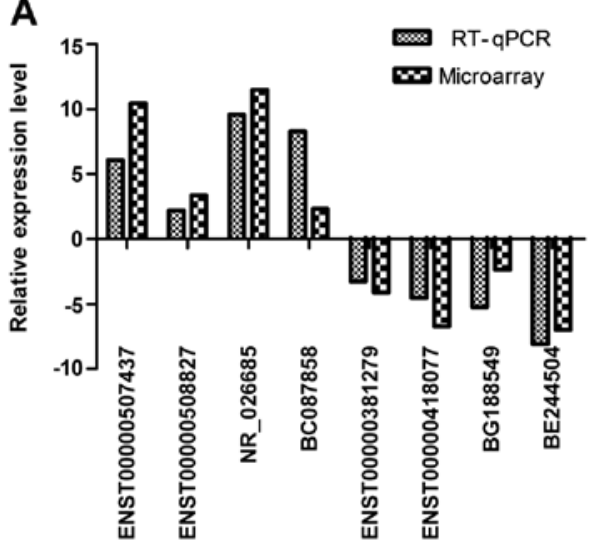

B

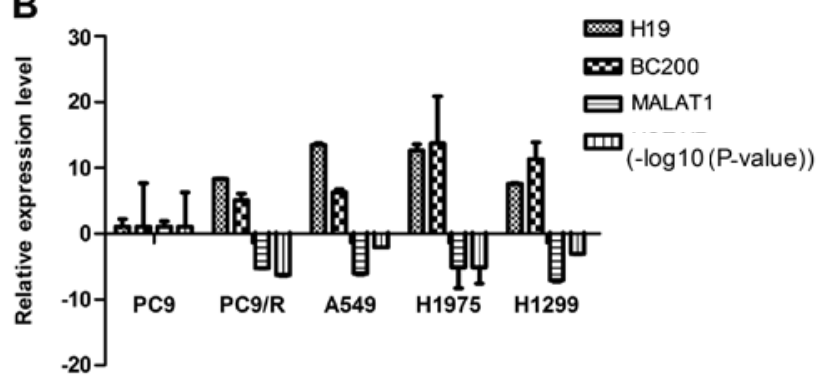

Figure 5. (A) Compared with the expression of lncRNAs using microarray and qRT-PCR. (B) Validating the expression of four studied lncRNAs by qRT-PCR in gefitinib-resistant lung cancer cells, compared with gefitinib-sensitive PC9 cells. P<0.01. lncRNAs, long non-coding RNAs.

tions) (Fig. 3A), while the downregulated transcripts were urogenital system development (GO: biological processes), extracellular space (GO: cellular components), and protein binding (GO: molecular functions) (Fig. 3B). Of note, the lower the P-value, the more significant the GO term (P-value cut-off of $\leq 0.05$ was recommended).We also found that 699 genes were associated with cell proliferation and cell apoptosis (Table II).

Pathway analysis. Based on the latest Kyoto Encyclopedia of Genes and Genomes (KEGG) database, we provided pathway analysis for differentially expressed mRNAs. This analysis allowed us to determine the biological pathway, which showed a significant enrichment of differentially expressed mRNAs.

The pathway analysis indicated that there were 19 pathways corresponding to the upregulated transcripts. The high-enrich- ment pathways targeted by overexpressed mRNAs were involved in RNA transport, lysosome and spliceosome (Fig. 4A). By contrast, there were 17 pathways involved in the downregulated transcripts. Significant pathways corresponding to downregulated mRNAs appeared to be responsible for salmonella infection, arachidonic acid metabolism, legionellosis, with the recommended P-value cut-off being 0.05) (Fig. 4B). Of these, the enriched-pathways associated with cell proliferation and apoptosis played a critical role in EGFR-TKIs resistance.

$R T-q P C R$ validation. We examined the expression of four studied lncRNAs (H19, BC200, MALAT1 and HOTAIR) in lung cancer cell lines using RT-qPCR to validate the results of IncRNA profiles of which $\mathrm{H} 19$ and BC200 were upregulated and MALAT1 and HOTAIR were downregulated. The 
resistance of cell lines to gefitinib was assessed using CCK-8 assay and results are shown in Fig. 1. Overexpression of H19 and BC200 were observed in gefitinib-resistant lung cancer cell lines (PC9/R, A549, H1299 and H1975). However, in the gefitinib-sensitive cell line (PC9), MALAT1 and HOTAIR were downregulated (Fig. 5B).

Four upregulated and four downregulated lncRNAs from differentially expressed lncRNAs were randomly selected. Using RT-qPCR the results of microarray in PC9/R vs. PC9 were validated. The expression levels of ENST00000507437, ENST00000508827, NR_026685 and BC087858 were upregulated and ENST00000381279, ENST00000418077, BG188549 and BE244504 were downregulated (Fig. 5A). Thus, the microarray data were confirmed by RT-qPCR.

\section{Discussion}

Aberrant lncRNAs expression patterns have been described in various types of cancer (10,20-22), and alterations in expression of lncRNAs correlate with prognosis and chemotherapy resistance of human cancer $(16,17)$. HOTAIR, HULC and $\mathrm{H} 19$ are crucial in the development and progression of tumors and are associated with chemotherapy resistance $(7,16,17)$. However, the functions of lncRNAs should be investigated in targeted therapy of human malignant tumors. In the present study, we showed that differentially expressed lncRNAs are associated with EGFR-TKIs resistance.

We first identified a total of 22,587 differentially expressed lncRNAs in gefitinib-sensitive and gefitinib-induced acquired-resistant lung cancer cells. Furthermore, to gain insight into the underlying biology of the differentially expressed transcripts, we utilized GO and pathway analyses to study the biological functions to these lncRNAs in the resistance of EGFR-TKIs. We found 699 genes associated with cell proliferation and apoptosis. In the pathway analysis, 19 pathways were identified to correspond to the upregulated transcripts. By contrast, there were 17 pathways involved in the downregulated transcripts. The enriched pathways associated with cell proliferation and apoptosis suggested involvement in EGFR-TKIs resistance. Previous studies reported that the mechanism of EGFR-TKIs resistance is associated with cell proliferation and cell apoptosis (23-25). Findings of our previous study showed that the activation of PI3K/Akt, MEK pathway and BIM were involved in the resistance of EGFRTKIs $(23,26)$. Those results indicate that some lncRNAs may play critical roles in gefitinib-resistant lung cancer cells through cell proliferation and apoptosis.

It has been reported that lncRNAs regulate the neighboring protein-coding genes (27). Therefore, we analyzed aberrant lncRNAs and their nearby coding genes. Some lncRNAs may play critical roles in regulating nearby genes that encode markers in EGFR-TKIs resistance. For example, upregulated lncRNAs-BC087858 is a 1,322 bp intergenic lncRNAs and was found to be located near forkhead box protein $\mathrm{C} 1$ (FOXC1), which is a member of the FOX transcription factor family and is important in cancer development (28). FOXC1 induces epithelial-mesenchymal transition (EMT) through inhibition of E-cadherin expression and promotes cell migration and invasion. Additionally, FOXC1 expression can be activated by epidermal growth factor/extracellular signal-related kinase (EGF/ERK) signaling pathways (29). It has been observed that NSCLC cells resistant to EGFR-TKIs exhibit EMT features (30-32). Thus, our results suggest that BC087858 may be involved in EGFRTKIs resistance through EMT. The lncRNA-RP11-15H7.2 is a $1,580 \mathrm{bp}$ intergenic lncRNAs that was found to be located near CITED2, a transcriptional modulator that is involved in human oncogenesis. Wu et al reported that CITED2 was involved in the resistance of cancer cells to the chemotherapeutic drug cisplatin (33). Therefore, lncRNAs may influence EGFR-TKIs resistance by regulating the nearby genes.

Additionally, we selected the expression of some studied IncRNAs (H19, HOTAIR, MALAT1 and BC200) to validate the consistency of microarray. Accumulating evidence suggests that H19 may be an oncogene or a tumor-suppressor gene that plays an important role in cancer development. Tsang and Kwok reported that $\mathrm{H} 19$ can induce MDR-1-associated chemotherapy resistance in human hepatocellular carcinoma cells (16). MALAT-1 was downregulated in gefitinib-resistant cells. In some studies, their potential roles of regulating EMT transcription have been reported (34). MALAT-1 was linked to EMT-associated transcription factors ZEB1, ZEB2, slug and E-cadherin. Moreover, MALAT-1 promoted EMT by activating the Wnt signaling pathway $(35,36)$. Our results suggested that those lncRNAs may be involved in the EGFR-TKIs resistance in NSCLC and may provide novel pathways for improved understanding of the molecular mechanisms underlying EGFR-TKIs resistance. However, future studies are required to fully elucidate the mechanisms by which lncRNAs may promote resistance to EGFR-TKIs.

In conclusion, we reported that numerous lncRNAs were differentially expressed between gefitinib-sensitive PC9 and gefitinib-resistant PC9/R cells, many of which played important roles in regulating EGFR-TKIs resistance through various mechanisms including EMT and cell apoptosis. It is suggested that lncRNAs can exert their functions through interactions with coding transcripts and proteins in NSCLC EGFR-TKIs resistance. However, the exact mechanisms of lncRNAs require further study and to determine whether the differentially expressed lncRNAs were involved in EGFR-TKIs resistance in NSCLC. Based on these results, future studies focusing on lncRNAs expression and functions should be performed to assist patients in the improvement of response to EGFR-TKIs.

\section{Acknowledgements}

This study was supported by grants from the National Natural Science Foundation of China (nos. 81372392 and 81172101), and the Key Project of the Science and Technology Commission of Shanghai Municipality (nos. 124119a800 and 11JC1411301).

\section{References}

1. Zhang Z, Lee JC, Lin L, et al: Activation of the AXL kinase causes resistance to EGFR-targeted therapy in lung cancer. Nat Genet 44: 852-860, 2012.

2. Zhou C, Wu YL, Chen G, et al: Erlotinib versus chemotherapy as first-line treatment for patients with advanced $E G F R$ mutationpositive non-small-cell lung cancer (OPTIMAL, CTONG-0802): a multicentre, open-label, randomised, phase 3 study. Lancet Oncol 12: 735-742, 2011.

3. Bar J and Onn A: Overcoming molecular mechanisms of resistance to first-generation epidermal growth factor receptor tyrosine kinase inhibitors. Clin Lung Cancer 13: 267-279, 2012. 
4. Turke AB, Zejnullahu K, Wu YL, et al: Preexistence and clonal selection of MET amplification in EGFR mutant NSCLC. Cancer Cell 17: 77-88, 2010

5. Bean J, Brennan C, Shih JY, et al: MET amplification occurs with or without T790M mutations in EGFR mutant lung tumors with acquired resistance to gefitinib or erlotinib. Proc Natl Acad Sci USA 104: 20932-20937, 2007.

6. Qiu M, Xu Y, Yang X, et al: CCAT2 is a lung adenocarcinomaspecific long non-coding RNA and promotes invasion of non-small cell lung cancer. Tumour Biol 35: 5375-5380, 2014

7. Liu Z, Sun M, Lu K, et al: The long noncoding RNA HOTAIR contributes to cisplatin resistance of human lung adenocarcinoma cells via downregualtion of $\mathrm{p} 21^{\mathrm{WAF} / \mathrm{CIP} 1}$ expression. PLoS One 8: e77293, 2013.

8. Gutschner T, Hämmerle M, Eissmann M, et al: The noncoding RNA MALATI is a critical regulator of the metastasis phenotype of lung cancer cells. Cancer Res 73: 1180-1189, 2013.

9. Liu XH, Liu ZL, Sun M, et al: The long non-coding RNA HOTAIR indicates a poor prognosis and promotes metastasis in non-small cell lung cancer. BMC Cancer 13: 464, 2013.

10. Takahashi K, Yan I, Haga $\mathrm{H}$ and Patel T: Long noncoding RNA in liver diseases. Hepatology 60: 744-753, 2014.

11. Zhao J and Lawless MW: Long noncoding RNAs and their role in the liver cancer axis. Nat Rev Gastroenterol Hepatol: Nov 19, 2013 (Epub ahead of print). doi: 10.1038/nrgastro.2013.87-c1.

12. Ren S, Wang F, Shen J, et al: Long non-coding RNA metastasis associated in lung adenocarcinoma transcript 1 derived miniRNA as a novel plasma-based biomarker for diagnosing prostate cancer. Eur J Cancer 49: 2949-2959, 2013.

13. Iacoangeli A, Lin Y, Morley EJ, et al: BC200 RNA in invasive and preinvasive breast cancer. Carcinogenesis 25: 2125-2133, 2004.

14. Svoboda M, Slyskova J, Schneiderova M, et al: HOTAIR long non-coding RNA is a negative prognostic factor not only in primary tumors, but also in the blood of colorectal cance patients. Carcinogenesis 35: 1510-1515, 2014

15. Yuan JH, Yang F, Wang F, et al: A long noncoding RNA activated by TGF- $\beta$ promotes the invasion-metastasis cascade in hepatocellular carcinoma. Cancer Cell 25: 666-681, 2014

16. Tsang WP and Kwok TT: Riboregulator H19 induction of MDRIassociated drug resistance in human hepatocellular carcinoma cells. Oncogene 26: 4877-4881, 2007.

17. Tsang WP, Wong TW, Cheung AH, et al: Induction of drug resistance and transformation in human cancer cells by the noncoding RNA CUDR. RNA 13: 890-898, 2007.

18. Jiang M,Huang O,Xie Z, et al: Anovel long non-coding RNA-ARA: adriamycin resistance-associated. Biochem Pharmacol 87: 254-283, 2014.

19. Yang Y, Li H, Hou S, et al: The noncoding RNA expression profile and the effect of lncRNA AK126698 on cisplatin resistance in non-small-cell lung cancer cell. PLoS One 8: e65309, 2013.

20. Gainor JF and Shaw AT: Emerging paradigms in the development of resistance to tyrosine kinase inhibitors in lung cancer. J Clin Oncol 31: 3987-3996, 2013.
21. Zhang EB, Yin DD, Sun M, et al: P53-regulated long non-coding RNA TUG1 affects cell proliferation in human non-small cell lung cancer, partly through epigenetically regulating HOXB7 expression. Cell Death Dis 5: e1243, 2014.

22. Hauptman N and Glavač D: Long non-coding RNA in cancer. Int J Mol Sci 14: 4655-4669, 2013.

23. Li H, Schmid-Bindert G, Wang D, et al: Blocking the PI3K/AKT and MEK/ERK signaling pathways can overcome gefitinibresistance in non-small cell lung cancer cell lines. Adv Med Sci 56: 275-284, 2011

24. Sordella R, Bell DW, Haber DA and Settleman J: Gefitinibsensitizing $E G F R$ mutations in lung cancer activate anti-apoptotic pathways. Science 305: 1163-1167, 2004.

25. Ng KP, Hillmer AM, Chuah CT, et al: A common BIM deletion polymorphism mediates intrinsic resistance and inferior responses to tyrosine kinase inhibitors in cancer. Nat Med 18 $521-528,2012$.

26. Li Z,Zhou S, Zhang L, et al: BIM induction of apoptosis triggered by EGFR-sensitive and resistance cell lines of non-small-cell lung cancer. Med Oncol 28: 572-577, 2011.

27. Yang F, Zhang L, Huo XS, et al: Long noncoding RNA high expression in hepatocellular carcinoma facilitates tumor growth through enhancer of zeste homolog 2 in humans. Hepatology 54: $1679-1689,2011$

28. Xia L, Huang W, Tian D, et al: Overexpression of forkhead box $\mathrm{C} 1$ promotes tumor metastasis and indicates poor prognosis in hepatocellular carcinoma. Hepatology 57: 610-624, 2013.

29. Wang J, Ray PS, Sim MS, et al: FOXC1 regulates the functions of human basal-like breast cancer cells by activating NF- $\kappa \mathrm{B}$ signaling. Oncogene 31: 4798-4802, 2012.

30. Uramoto H, Iwata T, Onitsuka T, et al: Epithelial-mesenchymal transition in EGFR-TKI acquired resistant lung adenocarcinoma. Anticancer Res 30: 2513-2517, 2010.

31. Bryant JL, Britson J, Balko JM, et al: A microRNA gene expression signature predicts response to erlotinib in epithelial cancer cell lines and targets EMT. Br J Cancer 106: 148-156, 2012.

32. Cufi S, Bonavia R, Vazquez-Martin A, et al: Silibinin suppresses EMT-driven erlotinib resistance by reversing the high $m i R$-21/low $m i R-200 c$ signature in vivo. Sci Rep 3: 2459, 2013.

33. Wu ZZ, Sun NK and Chao CC: Knockdown of CITED2 using short-hairpin RNA sensitizes cancer cells to cisplatin through stabilization of $\mathrm{p} 53$ and enhancement of p53-dependent apoptosis. J Cell Physiol 226: 2415-2428, 2011.

34. Samatov TR, Tonevitsky AG and Schumacher U: Epithelialmesenchymal transition: focus on metastatic cascade, alternative splicing, non-coding RNAs and modulating compounds. Mol Cancer 12: 107, 2013.

35. Ying L, Chen Q, Wang Y, et al: Upregulated MALAT-1 contributes to bladder cancer cell migration by inducing epithelial-to-mesenchymal transition. Mol Biosyst 8: 2289-2294, 2012.

36. Sequist LV, Waltman BA, Dias-Santagata D, et al: Genotypic and histological evolution of lung cancers acquiring resistance to EGFR inhibitors. Sci Transl Med 3: 75ra26, 2011. 